\title{
Calidad de los servicios de anticoncepción en El Alto, Bolivia
}

\author{
Carmen Velasco, ${ }^{1}$ Claudia de la Quintana, ${ }^{1}$ Gretzel Jové, ${ }^{1}$ \\ Luz Ángela Torres ${ }^{2}$ y Patricia Bailey ${ }^{3}$
}

RESUMEN El presente estudio tuvo por objetivo evaluar la calidad de los servicios de anticoncepción en la ciudad de El Alto, Bolivia. En su diseño se han contemplado cuatro elementos: 1) las relaciones entre los proveedores de servicios y sus clientes, 2) la disponibilidad de métodos anticonceptivos, 3) las condiciones de los servicios, y 4) la satisfacción de las usuarias. También se han tenido en cuenta las opiniones de los proveedores y de las usuarias y no usuarias de estos servicios, quienes se clasificaron como gubernamentales o no gubernamentales, de acuerdo con la administración de la institución a la que pertenecían. Los datos provinieron de un análisis de la situación de dichos servicios y de testimonios obtenidos de las participantes durante 1995. En cuanto a las relaciones interpersonales, se encontró que los proveedores percibían el trato del médico más favorablemente que las clientas, en tanto que las no usuarias lo percibían desfavorablemente. La percepción de un trato igualitario se correlacionó positivamente con la vestimenta que usaban las clientas. En cuanto a la disponibilidad de los métodos anticonceptivos, 15 de las 36 instituciones encuestadas no disponían de métodos modernos, a pesar de la existencia de una política nacional para proveerlos a la población. La oferta de estos servicios a parejas y a adolescentes es escasa, principalmente en las instituciones gubernamentales. El análisis de las condiciones de los servicios demostró que en algunas instituciones había problemas graves en la provisión de una atención de mínima calidad. Finalmente, este trabajo describe cómo la mayoría de estas limitaciones en la prestación de servicios de anticoncepción en El Alto pueden subsanarse mediante estrategias de costo moderado.

El marco conceptual que Bruce (1) y Jain (2) formalizaron sobre la calidad de los servicios de anticoncepción ha estimulado a los proveedores y a las agencias internacionales que trabajan en servicios de salud sexual y reproductiva (SSR) a medir y mejorar la calidad de dichos servicios. Una mujer

\footnotetext{
1 Programas para la Mujer (PRO MUJER), La Paz, Bolivia. Toda correspondencia debe dirigirse a esta autora a la siguiente dirección postal: Avenida 20 de Octubre, No. 2668, La Paz, Bolivia.

2 Hospital Universitario del Valle, Cali, Colombia.

3 Family Health International, Research Triangle Park, Carolina del Norte, Estados Unidos de América.
}

que posee información puede controlar su fecundidad y, consiguientemente, tomar decisiones apropiadas en otros aspectos de su vida. A su vez, un incremento de la satisfacción de las clientes puede redundar en mayores coberturas, y ello a su vez influir en el uso de los recursos con incrementos mínimos en los presupuestos de los programas (3). Con las metas reproductivas cumplidas, también se reducirían las tasas de embarazos no deseados y de mortalidad materna $(1,4,5)$.

Durante el proceso de integrar el concepto de calidad en los servicios de SSR de Bolivia, se han llevado a cabo diversos estudios que demostraron una baja utilización de los servicios y una preferencia por la medicina y los métodos anticonceptivos tradicionales (6-9). Esto se atribuye, entre otras causas, a la ausencia de educación sexual, a la poca oferta de servicios de SSR, a la desconfianza de la población con respecto a los servicios y los métodos, y a factores económicos y socioculturales $(7,8,10,11)$.

La ciudad de El Alto se encuentra situada al oeste de La Paz, en el altiplano boliviano. La tasa de crecimiento poblacional es de $9 \%$, debido principalmente a la migración desde las zonas rurales del departamento de La Paz, que le confiere una pluralidad 
de culturas (12). La tasa global de fecundidad es de 4,5 y la prevalencia del uso de métodos anticonceptivos llega a $51 \%$ en mujeres en edad de procrear. Doce por ciento de las mujeres utilizan métodos anticonceptivos modernos, tales como dispositivos intrauterinos, anticonceptivos orales o inyectables o la esterilización, en tanto que 39\% usan los métodos tradicionales como el ritmo y el retiro preeyaculatorio (13). A pesar de ello, un gran porcentaje de los embarazos no fueron deseados en el momento en que ocurrieron y el aborto inducido causa de 27 a $35 \%$ de las muertes maternas $(10,14)$.

En 1995, PRO MUJER (organización no gubernamental que ofrece capacitación y crédito a las mujeres), con el apoyo del Fondo de Población de las Naciones Unidas y de Family Health International, llevó a cabo un estudio que tuvo los siguientes objetivos: 1) determinar las necesidades de información y prestación de servicios de salud reproductiva de la población migrante de El Alto, y 2) proporcionar información acerca de la situación de todos los servicios de salud reproductiva y de las farmacias en El Alto. La investigación mostró que en esta ciudad existen condiciones estructurales y socioeconómicas que en ocasiones crean barreras para el acceso de la población migrante a los servicios de SSR $(12,15)$.

El presente trabajo ha tomado como base dicho estudio y se ha enfocado en cuatro aspectos de la calidad de los servicios de SSR en El Alto: 1) las relaciones interpersonales, 2) la disponibilidad de métodos anticonceptivos, 3) las condiciones de los servicios, y 4) la satisfacción de las usuarias. Estos aspectos se han examinado desde la perspectiva de los proveedores, de las usuarias y de las no usuarias de servicios, con el fin de conocer y comparar sus opiniones sobre la atención.

\section{MATERIALES Y MÉTODOS}

Se utilizaron dos estrategias para la recolección de datos. En primer lugar, se llevó a cabo un análisis de la situa- ción de 46 servicios, que constituyen casi la totalidad de los servicios de SSR en El Alto. Se emplearon los cinco instrumentos siguientes en este componente:

1. Encuesta a 36 directores de servicios que prestan atención en SSR (17 servicios gubernamentales y 19 no gubernamentales) 4 .

2. Encuesta a 85 proveedores de anticoncepción en los servicios de salud.

3. Encuesta de salida a 217 usuarias que asistieron al servicio de salud.

4. Encuesta a 215 no usuarias de servicios (personas que nunca habían asistido a un centro de salud por motivos de SSR).

5. Inventario infraestructural y de los medicamentos disponibles en 46 servicios de SSR.

La segunda estrategia consistió en la creación de 18 grupos focales con varones y mujeres de la población migrante, tanto en su sitio de origen como en su nuevo lugar de residencia. Durante las sesiones de los grupos focales se discutió lo que pensaba la población acerca de las ventajas y desventajas de vivir en zonas rurales o urbanas, salud sexual y reproductiva, anticoncepción, aborto y atención en los servicios de salud. Los testimonios derivados de los grupos focales urbanos se utilizaron para reforzar la información del análisis de la situación.

La recolección de datos se realizó de marzo a septiembre de 1995. Las guías para los grupos focales y las boletas, que fueron diseñadas con preguntas cerradas y precodificadas, fueron validadas por el equipo de investigación de PRO MUJER. Los perfiles socioeconómicos de los entrevistadores, varones y mujeres, fueron similares a los de los entrevistados, ya que aquellos incluso sabían hablar aymara. Las entrevistas se efectuaron según el criterio del sexo, de tal manera que los entrevistadores

\footnotetext{
4 Cuando el director era el proveedor de los servicios, se aplicó solo la encuesta de los proveedores, de manera que se entrevistó únicamente a 36 personas en calidad de directores.
}

varones trabajaron con varones y las mujeres con mujeres. Todos los participantes dieron su consentimiento verbal antes de ser entrevistados.

Cerca de $34 \%$ de los usuarios y $30 \%$ de los no usuarios eran varones. En este análisis no se hizo distinción entre los sexos y, como la mayoría de las entrevistadas eran mujeres, se hablará de usuarias y no usuarias. La comparación entre el grupo de usuarias y no usuarias reveló que un mayor número de las segundas usaban pollera (traje típico de la mujer del altiplano boliviano, indicador de raíces étnicas y culturales), tenían niveles de instrucción más bajos (definidos según los porcentajes que habían completado la enseñanza básica y la enseñanza superior) y habían emigrado más recientemente que las usuarias. Treinta y cinco por ciento de las usuarias y $54 \%$ de las no usuarias habían vivido de 1 a 5 años en El Alto y $65 \%$ habían vivido en esa localidad más de 5 años.

La comparación entre los proveedores y sus clientes y los clientes potenciales también identificó diferencias. En tanto que los proveedores procedían de la misma extracción socioeconómica que la población que atendían, no todos hablan aymara. Algunos de ellos establecen serias diferencias entre el saber médico y la presunta ignorancia e idiosincracia de la población a la que atienden. Dentro de los servicios existen relaciones de género desiguales, pues las auxiliares de enfermería tienen mayor contacto con la población atendida.

Para evaluar las condiciones de los servicios desde el punto de vista de las usuarias, se hicieron preguntas acerca de cuatro cualidades que debe tener un consultorio, así como la cualidad contraria (limpio o sucio; cómodo o incómodo; templado o frío; iluminado u oscuro). A cada par se le asignó un punto positivo o negativo, según correspondiera el caso.

El manejo y análisis de los datos se realizaron con el programa Epi Info versión 6.0 (16) y como prueba estadística se usó ji al cuadrado. El valor $P$, cuando menor de 0,05 , indicó un hallazgo estadísticamente significativo. 


\section{RESULTADOS}

En el cuadro 1 se muestra la distribución del personal que trabaja en los programas de SSR, según su profesión. Se observa que en ambos tipos de servicios el mayor número de proveedores está constituido por auxiliares de enfermería y ginecólogos, seguidos por los médicos generales.

\section{Relaciones interpersonales}

En el cuadro 2 se muestran tres aspectos de la percepción que tienen los proveedores, las usuarias y las personas no usuarias de SSR acerca de la interacción entre los proveedores y las usuarias de dichos servicios. Ochenta y tres por ciento de los proveedores en los consultorios gubernamentales opinaron que los médicos trataban bien a las clientes, a diferencia de $98 \%$ de los proveedores de los consultorios no gubernamentales. Entre las usuarias, 57\% de las que asistían a un centro guberna-
CUADRO 1. Personal que trabaja en los servicios de salud sexual y reproductiva, según el tipo de servicio. El Alto, Bolivia, 1994

\begin{tabular}{lcc}
\hline & \multicolumn{2}{c}{ Tipo de servicio } \\
\cline { 2 - 3 } \multicolumn{1}{c}{ Profesión } & $\begin{array}{c}\text { Gubernamental } \\
(n=17)\end{array}$ & $\begin{array}{c}\text { No } \\
\text { gubernamental } \\
(n=19)\end{array}$ \\
\hline Auxiliar de & & \\
enfermería & 6 & 13 \\
Ginecólogo & 4 & 6 \\
Médico general & 3 & 3 \\
Enfermera & 1 & 1 \\
Otro & 3 & 2 \\
\hline
\end{tabular}

mental y 75\% de las que asistían a un centro no gubernamental afirmaron que los médicos las trataban bien. Solamente $12 \%$ de las no usuarias percibieron que los médicos trataban bien a sus clientes. Casi todos los proveedores en ambos tipos de servicios afirmaron que daban las debidas explicaciones (sobre la manera en que procedería el médico) antes de examinar a la usuaria, pero solo un promedio de $75 \%$ de las usuarias refirieron haberlas recibido.

Entre 64 y 72\% de las usuarias se sentían atendidas sin discriminación y las restantes se sentían discriminadas. Entre las no usuarias, cerca de la mitad $(48 \%)$ opinaban que en los servicios no trataban por igual a todas las usuarias (cuadro 2). Las razones que explicaban esta desigualdad, según un tercio de las usuarias y aproximadamente la mitad de las no usuarias, se relacionaban con el "uso de pollera, la falta de dinero, o la discriminación". Una usuaria respondió: "Porque hay gente que a veces es un poquito pobre, no se va bien vestida ... Entonces no hay esa igualdad. Hay otra señora que va mejor vestida, aunque tenga los mismos derechos, siempre hay eso."

Dadas estas relaciones, se examinaron las experiencias o las percepciones según la vestimenta de las usuarias y no usuarias y se observó que en los servicios gubernamentales había una diferencia significativa entre la vesti-

CUADRO 2. Calidad de las relaciones entre el personal y las usuarias, según los proveedores de servicios de salud reproductiva, las usuarias y las no usuarias, por tipo de servicio. El Alto, Bolivia, 1994

\begin{tabular}{|c|c|c|c|c|c|}
\hline & \multicolumn{2}{|c|}{ Proveedores } & \multicolumn{2}{|c|}{ Usuarias } & \multirow[b]{2}{*}{ No usuarias } \\
\hline & \multicolumn{2}{|c|}{ Tipo de servicio } & \multicolumn{2}{|c|}{ Tipo de servicio } & \\
\hline & $\begin{array}{l}\text { Gubernamental } \\
\qquad \begin{array}{c}n=36) \\
\%\end{array}\end{array}$ & $\begin{array}{c}\text { No gubernamental } \\
\qquad \begin{array}{c}(n=49) \\
\%\end{array}\end{array}$ & $\begin{array}{c}\text { Gubernamental } \\
\qquad \begin{array}{c}n=99) \\
\%\end{array}\end{array}$ & $\begin{array}{c}\text { No gubernamental } \\
\left(\begin{array}{c}n=118) \\
\%\end{array}\right.\end{array}$ & $\begin{array}{c}(n=215) \\
\%\end{array}$ \\
\hline \multicolumn{6}{|l|}{ Trato dado por el médico } \\
\hline Bueno & 83 & 98 & 57 & 75 & 12 \\
\hline Regular & 17 & 2 & 39 & 24 & 52 \\
\hline Malo & 0 & 0 & 4 & 1 & 22 \\
\hline No sabía/no respondió & 0 & 0 & 0 & 0 & 14 \\
\hline Valor $P$ & \multicolumn{2}{|r|}{0,01} & \multicolumn{2}{|r|}{0,01} & \\
\hline \multicolumn{6}{|l|}{$\begin{array}{l}\text { A la usuaria se le dan } \\
\text { explicaciones antes del } \\
\text { examen físico. }\end{array}$} \\
\hline Sí & 100 & 98 & 72 & 77 & \\
\hline No & 0 & 2 & 28 & 23 & \\
\hline Valor $P$ & \multicolumn{2}{|r|}{0,39} & \multicolumn{2}{|r|}{0,40} & \\
\hline \multicolumn{6}{|c|}{$\begin{array}{l}\text { Las clientes son atendidas sin } \\
\text { discriminación. }\end{array}$} \\
\hline Sí & & & 64 & 72 & 17 \\
\hline No & & & 9 & 3 & 48 \\
\hline No sabía/no respondió & & & 27 & 25 & 35 \\
\hline Valor $P$ & & & \multicolumn{2}{|r|}{0,18} & \\
\hline
\end{tabular}


CUADRO 3. Experiencia o percepción de discriminación entre las usuarias y no usuarias, según su vestimenta, por tipo de servicio. El Alto, Bolivia, 1994

\begin{tabular}{|c|c|c|c|c|c|c|}
\hline & \multicolumn{4}{|c|}{$\begin{array}{c}\text { Usuarias } \\
\text { Tipo de servicio }\end{array}$} & & \\
\hline & \multicolumn{2}{|c|}{ Gubernamental } & \multicolumn{2}{|c|}{ No gubernamental } & \multicolumn{2}{|c|}{ No usuarias } \\
\hline \multicolumn{7}{|c|}{$\begin{array}{l}\text { La atendieron igual que a las otras } \\
\text { clientes. }\end{array}$} \\
\hline Sí & 54 & 76 & 65 & 80 & 15 & 28 \\
\hline No/No sabía & 46 & 24 & 35 & 20 & 85 & 72 \\
\hline Valor $P$ & \multicolumn{2}{|c|}{0,02} & \multicolumn{2}{|c|}{0,07} & \multicolumn{2}{|c|}{0,04} \\
\hline
\end{tabular}

a Traje típico de las mujeres del altiplano boliviano.

menta y la igualdad del trato percibida. Quienes usaban vestido sentían con más frecuencia que el trato era igualitario, a diferencia de las que usaban pollera. Las no usuarias tenían una percepción del trato aun más desfavorable que las usuarias, pero se observó la misma tendencia: quien usaba vestido percibía menos discriminación que quien usaba pollera (cuadro 3).

Respecto de la facilidad y comodidad con que las clientes podían hacer preguntas a los médicos durante la consulta, 73\% de ellas no indicaron estos problemas al hacer preguntas. De las usuarias que hicieron preguntas durante su consulta, 98\% recibieron respuesta; 79\% dijeron haberla entendido, y las restantes entendieron muy poco o nada de lo explicado por el proveedor.

Los relatos de dos usuarias revelaron experiencias muy disintas con los servicios de salud: "Dan algún examen e no endecan de qué adolece. Muchas veces se le pregunta, se le dece: - ¿qué es lo que tengo?, - ¿para qué querés saber?" La respuesta es esa ¿no? "Yo voy ande él aun cuando es lejos, yo prefiero. Porque él me tiene cariño, me habla, me explica, me . . . ¿yo le explico no? Todo. Lo que no entiendo, no sé, yo le pregunto y él todo me responde, me orienta. Y bajo ese médico yo me ... yo no tengo ya más hijos; tiene mi hijo el ultimito ya 10 años y yo no me he hecho curar con nada, solamente me cuido".

\section{Disponibilidad de información y de métodos anticonceptivos}

De los proveedores, $96 \%$ informaron que sus clientes solicitaban información sobre la anticoncepción y que $78 \%$ de las usuarias, en el momento de recibir la orientación, deseaban usar algún método anticonceptivo. Para poder satisfacer esta demanda, $84 \%$ de los proveedores afirmaron disponer de tiempo suficiente y $60 \%$ dijeron poseer el material audiovisual de apoyo necesario para brindar una información adecuada. Según las usuarias, se les enseñaban los métodos de anticoncepción en $13 \%$ de las consultas.

De los directores de SRR, 64\% dijeron que siempre se satisfacía la demanda de métodos reversibles y modernos de anticoncepción en los servicios, en tanto que los restantes la consideraban insuficiente. Seis servicios gubernamentales y nueve no gubernamentales no tenían disponible ningún método anticonceptivo moderno. Cuatro de los servicios no gubernamentales disponían de cinco métodos (dispositivo intrauterino, píldora, producto inyectable, condón y espermicida), mientras que ningún consultorio gubernamental los tenía. Once centros no contaban con el material necesario para la inserción del dispositivo intrauterino. La esterilización quirúrgica solo se ofrecía en los dos hospitales gubernamentales. De los 19 consultorios no gubernamentales, tres eran administrados por religiosos y no tenían disponible ningún tipo de anticonceptivo moderno. Casi todos los servicios ofrecían información sobre el ritmo, pero pocos promovían los otros métodos naturales (cuadro 4).

Aunque las normas del Ministerio de Salud no exigen la autorización de la pareja para proveer un método anticonceptivo, ${ }^{5} 70 \%$ de los proveedores indicaron haberla solicitado siempre o en alguna ocasión. De 26 usuarias que solicitaron la autorización de sus cónyuges para usar un método, 23 la solicitaron para la esterilización quirúrgica, una para el DIU y dos, para otros métodos.

Respecto de la presencia de barreras que limitan el acceso a la elección del método, se encontró que 13 de los 17 servicios gubernamentales y 11 de los 19 no gubernamentales brindaban servicios de asesoramiento y orientación a adultos que asistían solos al centro. Dos directores de ambos tipos de centros (cuatro en total) desconocían este dato. Solo cinco de las instituciones no gubernamentales indicaron brindar

\footnotetext{
5 En el caso de la esterilización voluntaria, la autorización del esposo se recomienda en las normas del Ministerio de Salud (17).
} 
CUADRO 4. Número de métodos anticonceptivos modernos disponibles, por tipo de servicio. El Alto, Bolivia, 1994

\begin{tabular}{ccc}
\hline & \multicolumn{2}{c}{ Tipo de servicio } \\
\cline { 2 - 3 } $\begin{array}{c}\text { Métodos anticonceptivos } \\
\text { modernos disponibles } \\
\text { (No.) }\end{array}$ & $\begin{array}{c}\text { Gubernamental } \\
(n=17)\end{array}$ & $\begin{array}{c}\text { No gubernamental } \\
(n=19)\end{array}$ \\
\hline 0 & 6 & 9 \\
1 & 1 & 1 \\
2 & 4 & 3 \\
3 & 5 & 2 \\
4 & 1 & 0 \\
5 & 0 & 4 \\
\hline
\end{tabular}

servicios de información y asesoramiento dirigidos a adolescentes, y ningún director de las instituciones gubernamentales indicó ofrecer o saber si se ofrecía dicho servicio a personas de este grupo de edad. Finalmente, más de la mitad de los directores en los dos tipos de servicios dijeron que no ofrecían el servicio a parejas (cuadro 5).

De los proveedores, $85 \%$ indicaron que prestaban atención a mujeres solas y $81 \%$, a hombres solos. Entre quienes no brindaban atención a individuos solos, la mayoría expresaron razones relacionadas con las "políticas o restricciones de la institución" o con el deseo de "proteger a la pareja y a la familia"; en el caso de auxiliares y enfermeras, se indicó que "es el médico quien presta el servicio directamente".

De las usuarias, $43 \%$ afirmaron que serían atendidas si asistían solas al servicio de SSR; $32 \%$ opinaban que su pareja sería atendida si asistiera sola al centro, y el resto pensaba que no recibiría atención o lo ignoraba. Un tercio de las no usuarias creían que serían atendidas si asistían solas al centro; $21 \%$ creían que su pareja sería atendida si asistiera sola, y 56\% no sabían.

\section{Condiciones de los servicios}

En esta sección se examinaron el tiempo que invertía la usuaria en la espera y la consulta, así como las características físicas de los consultorios. Como se observa en el cuadro 6, en ambos servicios el proveedor notificó una espera menor que la indicada por la usuaria. En promedio, $14 \%$ de los proveedores indicaron 60 minutos de espera o más, en comparación con $29 \%$ de las usuarias en los servicios gubernamentales y $21 \%$ en los no gubernamentales. En cuanto al tiempo de consulta, $67 \%$ de los proveedores en los servicios gubernamentales y $63 \%$ de los proveedores en los servicios no gubernamentales estimaban la duración de sus consultas en más de 11 minutos;

CUADRO 5. Oferta de información y asesoramiento sobre anticoncepción para distintos tipos de destinatarios, según los directores de los servicios. El Alto, Bolivia, 1994

\begin{tabular}{|c|c|c|c|c|c|c|}
\hline \multirow[b]{4}{*}{ Destinatarios } & \multicolumn{6}{|c|}{ Oferta de información y asesoramiento } \\
\hline & \multicolumn{6}{|c|}{ Tipo de servicio } \\
\hline & \multicolumn{3}{|c|}{$\begin{array}{l}\text { Gubernamental } \\
\qquad(n=17)\end{array}$} & \multicolumn{3}{|c|}{$\begin{array}{l}\text { No gubernamental } \\
\qquad(n=19)\end{array}$} \\
\hline & Sí & No & No sabía & Sí & No & No sabía \\
\hline Adultos solos & 13 & 2 & 2 & 11 & 6 & 2 \\
\hline Adolescentes & 0 & 11 & 6 & 5 & 12 & 2 \\
\hline Parejas & 3 & 10 & 4 & 4 & 12 & 3 \\
\hline
\end{tabular}

en cambio, $43 \%$ de las usuarias de los servicios gubernamentales y $51 \%$ de las usuarias de los servicios no gubernamentales indicaron que las consultas duraban más de 11 minutos.

Se observó una relación entre el tiempo de espera y la satisfacción con el servicio. Setenta y seis por ciento de las usuarias que esperaron menos de 45 minutos se sentían satisfechas. No se sentían satisfechas $45 \%$ de las que habían esperado más de una hora. Una usuaria insatisfecha comentó: "En cuando aquí también, igual es en las postas, uno va a las postas, igual nos hacen esperar, el médico no llega, dicen que va a llegar más tarde. .."

Todas las instalaciones no gubernamentales contaban con agua potable y electricidad. De los 17 servicios gubernamentales, cinco no disponían de agua potable y dos no disponían de electricidad. Solo tres de los servicios gubernamentales y cuatro de los no gubernamentales contaban con más de tres consultorios para la provisión confidencial de información y atención a los clientes. En los siguientes testimonios se aprecia la impresión de las usuarias en relación con la confidencialidad del asesoramiento: "Casi no hay, no está, no hay comodidad, privacidad peor... A no ser que sea particular, pienso, ¿no? Pero nunca me ha ocurrido, nunca he ido". "Sí, da miedo conversar con el médico a veces, porque no hay un lugar adecuado para conversar, contar de nuestros problemas, de nuestras enfermedades, ¿no?"

El cuadro 7 muestra las condiciones de los consultorios, según las usuarias. Pocas encontraron servicios que fueran al mismo tiempo limpios, cómodos, templados e iluminados (puntaje $=4$ ), y en este sentido no se detectaron diferencias significativas entre los servicios gubernamentales y los demás. Se desprende de los siguientes comentarios que algunas usuarias reconocían las fallas de los servicios, independientemente, en ocasiones, de la competencia técnica del proveedor: "Tenemos una posta ... que poco a poco se ha estado equipando ¿no? Porque en años anteriores estaba bastante desequipado". "Los lugares que yo generalmente he visitado es muy frío ¿no? No hay una atención bien 
CUADRO 6. Percepción de los tiempos de espera y consulta para cada proveedor según las usuarias, por tipo de servicio. El Alto, Bolivia, 1994

\begin{tabular}{|c|c|c|c|c|}
\hline \multirow[b]{3}{*}{$\begin{array}{l}\text { Tiempo } \\
\text { (minutos) }\end{array}$} & \multicolumn{2}{|c|}{ Proveedor } & \multicolumn{2}{|c|}{ Usuaria } \\
\hline & \multicolumn{2}{|c|}{ Tipo de servicio } & \multicolumn{2}{|c|}{ Tipo de servicio } \\
\hline & $\begin{array}{l}\text { Gubernamental } \\
\qquad \begin{array}{c}n=36) \\
\%\end{array}\end{array}$ & $\begin{array}{l}\text { No gubernamental } \\
\qquad \begin{array}{c}(n=49) \\
\%\end{array}\end{array}$ & $\begin{array}{l}\text { Gubernamental } \\
\qquad \begin{array}{c}n=99) \\
\%\end{array}\end{array}$ & $\begin{array}{c}\text { No gubernamental } \\
\qquad \begin{array}{c}(n=118) \\
\%\end{array}\end{array}$ \\
\hline \multicolumn{5}{|l|}{ De espera } \\
\hline $15-30$ & 53 & 61 & 43 & 46 \\
\hline $31-59$ & 28 & 31 & 28 & 33 \\
\hline $60+$ & 19 & 8 & 29 & 21 \\
\hline Valor $P$ & \multicolumn{2}{|c|}{0,31} & \multicolumn{2}{|c|}{0,38} \\
\hline \multicolumn{5}{|l|}{ De consulta } \\
\hline 0-10 & 33 & 37 & 57 & 49 \\
\hline $11-15$ & 45 & 26 & 26 & 36 \\
\hline$>15$ & 22 & 37 & 17 & 15 \\
\hline Valor $P$ & \multicolumn{2}{|c|}{0,18} & \multicolumn{2}{|c|}{0,23} \\
\hline
\end{tabular}

¿no?" "El médico es bastante bueno, también capacitado, pero eh, carecía de material más que todo ese centro ¿no?"

\section{Satisfacción de las usuarias}

Finalmente, $86 \%$ de las usuarias indicaron sentirse satisfechas con la atención. Cerca de la mitad comentó "trato amable, buena orientación; comprensión; examinan bien; confianza, rapidez, cuidado", y un tercio comentó acerca del proveedor, "buen profesio-

CUADRO 7. Puntaje total asignado a los consultorios por las usuarias según sus características positivas y negativas, por tipo de servicio. El Alto, Bolivia, 1994

\begin{tabular}{|c|c|c|}
\hline \multirow[b]{2}{*}{ Puntos ${ }^{a}$} & \multicolumn{2}{|c|}{ Tipo de servicio } \\
\hline & $\begin{array}{l}\text { Gubernamental } \\
\qquad \begin{array}{c}n=87) \\
\%\end{array}\end{array}$ & $\begin{array}{c}\text { No } \\
\text { gubernamental } \\
(n=114) \\
\%\end{array}$ \\
\hline$<0$ & 19 & 12 \\
\hline $0-2$ & 65 & 60 \\
\hline $3-4$ & 16 & 28 \\
\hline Valor $P$ & \multicolumn{2}{|c|}{0,07} \\
\hline $\begin{array}{l}\text { El puntaje } \\
\text { rias asign } \\
\text { rísticas p } \\
\text { pio, cómo } \\
\text { rubro = } \\
\text { frío, oscu }\end{array}$ & $\begin{array}{l}\text { lo es la suma de los } p \\
\text { cada consultorio en vi } \\
\text { y negativas: caracterí } \\
\text { iplado, iluminado (pu } \\
\text { acterísticas negativas } \\
\text { os que vale cada rubr }\end{array}$ & $\begin{array}{l}\text { untos que las usua- } \\
\text { rtud de sus caracte- } \\
\text { sticas positivas: lim- } \\
\text { ntos que vale cada } \\
\text { : sucio, incómodo, } \\
(0=-1) \text {. }\end{array}$ \\
\hline
\end{tabular}

nal, contestó, explicó bien, sostuvo charla fácil de entender, tranquiliza y anima". Otros aspectos señalados fueron "medicamentos gratuitos; buenas recetas, buenos medicamentos y consejos, dieron ayuda oportuna, ayudan a los necesitados". Los comentarios de las usuarias que no estaban satisfechas (14\%) fueron "mala atención, no examinan, tardan demasiado, no dan un diagnóstico claro, reniegan de todo, solamente extienden recetas, no orientan".

\section{Problemas y soluciones}

Los directores y proveedores de SSR manifestaron su opinión acerca de los problemas existentes y dieron recomendaciones para mejorar la calidad de los servicios. De los directores, aproximadamente $75 \%$ identificaron como problemas la limitada oferta y promoción de servicios, la falta de equipo y personal, y defectos de la calidad de la atención ("maltrato a los usuarios"). Otros aspectos notificados se relacionaron con los rasgos socioeconómicos y culturales de la población (falta de recursos económicos, población heterogénea). Según $60 \%$ de los proveedores, la poca demanda de servicios se debía a la poca difusión de información sobre los servicios, a la carestía de los servicios y a la falta de equipo. Pocos proveedores identificaron la mala atención como un problema (1\%).

Como posibles soluciones, $40 \%$ de los directores sugirieron mejorar la infraestructura física y los equipos; $17 \%$ sugirieron dar un trato humanizado. Hubo sugerencias adicionales, principalmente realizar actividades de promoción y educación y adecuar el costo de los servicios. Más de $80 \%$ de los proveedores propusieron incrementar las actividades de información y promoción de los SSR, mejorar la infraestructura y los equipos y ofrecer mayor capacitación. Mejorar el trato a las clientes fue una recomendación de solo 6\% de los proveedores. Los demás sugirieron reducir los costos y ubicar los servicios en lugares más apropiados.

Más de la mitad de las usuarias y $82 \%$ de las no usuarias recomendaron mejorar los servicios y atraer clientes nuevos. Estas recomendaciones se relacionaron directamente con la calidad de la atención en términos de rapidez, confiabilidad, amabilidad de los profesionales, sentido de la ética y cumplimiento de los horarios. Cuarenta y un por ciento de las usuarias y $36 \%$ de las no usuarias deseaban mejorar la infraestructura y los equipos (tener ambientes amplios, mejor iluminados $\mathrm{y}$ con calefacción). Otras propusieron promover los servicios, disminuir los costos y combinar la medicina occidental y la tradicional.

\section{DISCUSIÓN}

Un aspecto del presente estudio que le confiere especial interés es la identificación de puntos de coincidencia entre los tres grupos de participantes: proveedores, usuarias y no usuarias. Dicho enfoque permitió, al mismo tiempo, identificar algunas prácticas y circunstancias que menoscaban la calidad de los servicios de SSR.

La calidad del trato interpersonal fue percibida de una manera muy distinta según el tipo de persona entrevistada: los proveedores se expresaron más favorablemente y las no usuarias fueron las que más criticaron los servicios. La percepción de discriminación 
en función de la clase socioeconómica o de la vestimenta, fundamentada o no, es la antítesis del trato humano y lleva a la poca utilización de los servicios y a la insatisfacción de la clientela. Se espera que los resultados de este estudio lleven a los proveedores a la reflexión y a cambiar su actitud frente a las usuarias, brindándoles un trato igualitario, cálido y humanizado, sin distinción de origen étnico, sexo o clase social.

La disponibilidad de métodos anticonceptivos y de información sobre ellos es un elemento esencial de cualquier servicio de anticoncepción. La demanda de orientación en materia de anticonceptivos es muy grande, pero el poco material informativo para el público suele ser usado. También hay escasez de anticonceptivos para distribución. El abastecimiento de los anticonceptivos se hace por mediación del Ministerio de Salud en el caso de los centros gubernamentales y de agencias de cooperación o entidades privadas en el caso de los otros centros. El Ministerio de Salud todavía prohíbe la distribución de anticonceptivos inyectables para el uso general. La provisión de otros anticonceptivos se realiza mensualmente o cada semestre; sin embargo, los resultados indican que su control es deficiente.

Las usuarias y no usuarias de SSR no tienen, en su mayoría, la certeza de ser atendidas si ellas o su pareja acuden solas a los centros. Este factor podría agravar su desconfianza o hacer que algunas clientes potenciales desistan de acudir en busca de asesoramiento por temor a ser rechazadas. Los proveedores, e indirectamente las usuarias, se ven afectados por algunas políticas institucionales que impiden brindar atención a personas solas y a los adolescentes. En lo que respecta a estos últimos, los servicios gubernamentales parecen carecer de una política de apoyo para asesorarlos sobre la adquisición de herramientas útiles para evitar comportamientos de riesgo que los exponen a los embarazos no planeados, a las enfermedades de transmisión sexual, a los abortos riesgosos y a otros problemas de salud sexual y reproductiva.

Los proveedores han eliminado algunas de las barreras que obstaculizaban el acceso a los servicios, particularmente la autorización de los esposos para la colocación de un DIU, para la cual solo una minoría de los proveedores exige un permiso. Esto constituye un logro para las mujeres que desean usar métodos anticonceptivos. Sin embargo, en una población donde la mayoría de las parejas tienen más hijos de los que desean, exigir la autorización del esposo para la esterilización quirúrgica voluntaria constituye una barrera para las mujeres que desean recurrir a ella.

Los servicios gubernamentales parecen tener condiciones más deficientes que los no gubernamentales. En los primeros hay mayor proporción de enfermeras auxiliares, menos insumos y menos consultorios en buenas condiciones. Los participantes de los tres grupos difirieron en sus opiniones y creencias sobre los defectos de los servicios. Por ejemplo, el tiempo de espera antes de la consulta fue mayor cuando opinó la usuaria y la duración de la consulta, cuando opinó el prove- edor. Si se considera que las usuarias son quienes más divulgan los servicios en la comunidad, sus experiencias desfavorables pueden llevar a otras usuarias y a mujeres que aún no son usuarias a no acudir a los SSR.

En muchos consultorios no hay suficiente espacio habilitado para dar asesoramiento confidencial. Esto podría llevar a la usuaria a no hacer preguntas abiertamente y a no despejar todas sus dudas cuando acude al proveedor, lo cual podría redundar en el uso inadecuado del método anticonceptivo y en una serie de experiencias desfavorables para la usuaria.

Algunos de los consultorios que carecen de agua potable y luz eléctrica están situados en el área urbana de la ciudad. La comunidad o el personal de salud debe acudir a los comités municipales para resolver los problemas que plantean las instalaciones eléctricas y de agua potable en los consultorios que carecen de ellas. Pese a que las usuarias no suelen estar en condición de juzgar la capacidad técnica de los proveedores, sí son capaces de discernir si la falta de atención o la atención de mala calidad se debe a la incompetencia de los proveedores o a la ausencia de equipo adecuado en los servicios.

La estrategia de cambiar la calidad de los servicios, como tarea constante, implica recorrer un camino muy largo. No obstante, a medida que los directores de los SSR se preocupen por evaluar sus servicios de manera constante y por tomar en cuenta tanto las opiniones de los proveedores como las de las usuarias y no usuarias de los servicios, las verdaderas necesidades de

\section{REFERENCIAS}

1. Bruce J. Fundamental elements of the quality of care: a simple framework. Stud Fam Plan 1990;21:61-91.

2. Jain AK. Managing quality of care in population programs. West Hartford, CT: Kumarian Press; 1992.

3. Hardee K, Gould BJ. A process for quality improvement in family planning services. Int Fam Planning Perspect 1992;19:147-152.
4. Bongaarts J, Parker Mauldin W, Phillips JF. The demographic impact of family planning programs. Stud Fam Plan 1990;21:299-310.

5. Jain, AK. Fertility reduction and the quality of family planning services. Stud Fam Plan 1989;20:1-16.

6. Rance S. Planificación familiar: se abre el debate. La Paz: Consejo Nacional de Población; 1990.
7. Bolivia, Ministerio de Desarrollo Humano. Diagnóstico cualitativo de la atención en salud reproductiva en Bolivia: revisión bibliográfica. La Paz: Secretaría Nacional de Salud; 1995. (Serie Expandiendo Opciones de Planificación Familiar).

8. Organización Mundial de la Salud. Diagnóstico cualitativo de la atención en salud reproductiva en Bolivia: investigaciones sobre la 
introducción y transferencia de tecnologías para la regulación de la fertilidad. Ginebra: Organización Mundial de la Salud; 1996. (Serie Expandiendo Opciones de Planificación Familiar).

9. The Johns Hopkins School of Hygiene and Public Health, Center for Communications Programs. La salud reproductiva está en tus manos: impacto de la campaña del Programa Nacional de Salud Reproductiva de Bolivia, 1996. Baltimore: JHSHPH; 1996.

10. Rance S, Olivares C. Mitos y realidades: el aborto en Bolivia. La Paz: Consejo de Población, Servicio de Información para el Desarrollo; 1995.

11. Rance S. Mujer, aborto y atención en salud: una historia de todos los días. La Paz: Secretaría Nacional de Salud; 1995.

12. Proyecto de Fortalecimiento de la Atención Primaria en el Distrito III de la ciudad de El
Alto, La Paz, Bolivia. Diagnóstico rápido, situación de la salud en el Distrito III, El Alto. La Paz: Riverijos Ltda; 1991.

13. Velasco C, De La Quintana C, Jové G. Salud reproductiva en población migrante: El Alto y áreas rurales, Departamento de La Paz. La Paz: Programas para la Mujer (PRO MUJER); 1996.

14. Instituto Nacional de Estadística. Encuesta Nacional de Demografía y Salud: informe departamental, Demographic and Health Surveys. La Paz: Macro International, Inc.; 1994.

15. Subcomisión de Investigación, Evaluación y Políticas de Población. Salud reproductiva en población migrante: El Alto y áreas rurales, Departamento de La Paz. Resumen de investigaciones actuales: población, desarrollo, salud sexual y reproductiva, 1996; 4(sept).

16. Dean AG, Dean JA, Coulombier D, Brendel KA, Smith DC, Burton AH, et al. Epi Info ver- sion 6: a word processing, database, and statistics program for epidemiology on microcomputers. Centers for Disease Control and Prevention, Atlanta, Georgia; 1994.

17. Bolivia, Secretaría Nacional de Salud y Johns Hopkins Program for International Education in Reproductive Health. Salud sexual y reproductiva (servicios, orientación, planificación familiar, introducción al manejo de enfermedades de transmisión sexual, investigación de la pareja infértil). Segunda edición. La Paz: Centro de Información para el Desarrollo; 1996.

Manuscrito recibido el 13 de febrero de 1998 y aceptado para publicación, tras revisión, el 12 de enero de 1999.

ABSTRACT The objective of this study was to evaluate the quality of contraception services in the city of El Alto, Bolivia. In the study design, four components were considered: 1) interpersonal relations between service providers and users, 2) the availability of vari-

\section{The quality of contraception services in El Alto, Bolivia} ous contraceptive methods, 3) conditions in the service centers, and 4) user satisfaction. The opinions of three groups were taken into account: service providers, service users, and nonusers. The service centers were classified as either governmental or nongovernmental, depending on the management of the institution to which the service center belonged. The study data came from a situation analysis of the services and from comments gathered from study participants in 1995. The study found that providers held a more favorable view than did service users of the interpersonal relations and personal treatment that physicians provided. Nonusers had an unfavorable perception of physicians' treatment of users. Users' perceptions of receiving egalitarian treatment correlated with their style of dress. With regard to the availability of contraceptive methods, 15 of the 36 centers surveyed did not have modern methods, despite there being a national policy to provide them to the public. The supply of contraception services for couples and for adolescents is limited, especially in the governmental institutions. The analysis of the conditions in the service centers demonstrated that some institutions had serious difficulties providing services of at least a minimum quality. Finally, the study describes how most of the service limitations in El Alto can be corrected through moderate-cost strategies. 